\title{
Treatment with metformin and a dipeptidyl peptidase-4 inhibitor elevates apelin levels in patients with type 2 diabetes mellitus
}

This article was published in the following Dove Press journal:

Drug Design, Development and Therapy

14 August 2015

Number of times this article has been viewed

\section{Yujuan Fan* \\ Yu Zhang* \\ Xuesong $\mathrm{Li}^{*}$ \\ Hui Zheng \\ Yuping Song \\ Ning Zhang \\ Chunfang Shen \\ Xiaofang Fan \\ Fengdong Ren \\ Jiayi Shen \\ Guoguang Ren \\ Jialin Yang}

Department of Endocrinology, Central Hospital of Minhang District, Minhang Hospital affiliated to Fudan University, Shanghai, People's Republic of China

*These authors contributed equally to this work
Correspondence: Jialin Yang Department of Endocrinology, Central Hospital of Minhang District, Minhang Hospital affiliated to Fudan University, Xinsong Road 170, Minhang District, Shanghai, People's Republic of China Tel +86 2I 64923400 ext 5 I52 Fax +86 2I 63353230 Email jialinyang2002@163.com
Background: The objective of this study was to assess the effects of metformin monotherapy or combined treatment with a dipeptidyl peptidase-4 inhibitor (vildagliptin) on apelin levels in patients with type 2 diabetes mellitus.

Methods: Twenty-five patients with poor glycemic control (glycosylated hemoglobin $>6.5 \%$ [48 mmol/mol]) taking 1,000 $\mathrm{mg}$ of metformin daily and 25 healthy controls matched for age and body mass index were enrolled in this study. Anthropometric parameters, glycemic and lipid profile, insulin resistance (homeostasis model assessment of insulin resistance index), and apelin levels were measured at baseline and at 12-week and 24-week visits.

Results: At baseline, apelin levels were higher in the T2DM patients than in the controls $(1.93 \pm 1.81 \mathrm{ng} / \mathrm{mL}$ versus $6.09 \pm 4.90 \mathrm{ng} / \mathrm{mL} ; P<0.05)$. After 12 weeks, when vildagliptin was added, fasting blood glucose and glycosylated hemoglobin decreased, and apelin levels increased further (from $6.09 \pm 4.90 \mathrm{ng} / \mathrm{mL}$ to $24.23 \pm 12.59 \mathrm{ng} / \mathrm{mL} ; P<0.05$ ). Follow-up at 24 weeks showed no further improvement in the glycemic profile and no further increase in apelin levels.

Conclusion: Both metformin and vildagliptin favorably changed glycemic indices and apelin levels. For patients inadequately controlled on a low dose of metformin, addition of vildagliptin may be helpful.

Keywords: glucagon-like peptides, glucose-dependent insulinotropic polypeptide, antidiabetic drug, adipocytokine

\section{Introduction}

Apelin is a novel adipocytokine produced by white adipose tissue and binds the APJ receptor with high affinity. ${ }^{1}$ Apelin and the APJ receptor are expressed in pancreatic islet cells, ${ }^{2}$ and appear to be involved in energy metabolism. Many studies show that apelin plays a role in the regulation of glucose homeostasis, glucose-stimulated insulin secretion, and even insulin sensitivity. ${ }^{3-7}$ These observations suggest that apelin has an important role in diabetes mellitus, not only as a therapeutic target, ${ }^{8}$ but also as a biomarker.

Studies have reported decreased circulating apelin levels in patients with newly diagnosed and untreated type 2 diabetes mellitus (T2DM). ${ }^{9}$ Our research group has also reported low plasma apelin levels in Chinese people with newly diagnosed T2DM. ${ }^{10}$ Although experimental research supports the effects of this adipocytokine on insulin resistance, clinical studies evaluating the differential effects of antidiabetic drugs on plasma levels of apelin are lacking. Both metformin and dipeptidyl peptidase (DPP)-4 inhibitors improve glycemic control ${ }^{11}$ and act on adipose tissue by improving the dysregulated "adipocytokine" profile in the insulin-resistant state. ${ }^{12,13}$ Therefore, studies on the effects of pharmaceutical interventions, especially metformin and DPP-4 inhibitors, on apelin levels are warranted. 


\section{Materials and methods Subjects and study design}

Twenty-five patients with T2DM and 25 healthy controls matched for age and body mass index (BMI) were enrolled in this study. Study inclusion criteria included already being treated with metformin $(1,000 \mathrm{mg} /$ day, Sino-American Shanghai Squibb Pharmaceuticals Ltd, Shanghai, People's Republic of China) alone for at least 4 weeks but without adequate glycemic control, ie, glycosylated hemoglobin $\left(\mathrm{HbA}_{1 \mathrm{c}}\right)>6.5 \%(48 \mathrm{mmol} / \mathrm{mol})$. None of the diabetic patients had acute or chronic complications. A DPP-4 inhibitor (vildagliptin $100 \mathrm{mg} /$ day, Novartis Europharm Ltd, Horsham, UK) was added for at least 24 weeks. No patient had clinical evidence of cardiovascular disease (coronary, peripheral, carotid artery) or any other major chronic disease (autoimmune, life-threatening). None of female patients had polycystic ovary syndrome. All participants were instructed not to change their dietary habits or daily activities during the study period. At the 12-week visit, the patients were excluded if their $\mathrm{HbA}_{1 \mathrm{c}}$ was $>9 \%$ and if they had received rescue therapy. All procedures were in accordance with the ethical standards of the responsible committee on human experimentation (institutional and national) and with the Declaration of Helsinki. This study was approved by the ethics committee of Minhang Hospital affiliated to Fudan University. Informed consent was obtained from all patients before inclusion in the study.

\section{Blood analyses}

Blood samples were collected between 8 am and 8.30 am after 12 hours of fasting. The tubes were promptly centrifuged, and the plasma was separated and stored at $-80^{\circ} \mathrm{C}$. All samples were run in the same assay. Plasma glucose, urea, creatinine, uric acid, total cholesterol, low-density lipoprotein cholesterol, high-density lipoprotein cholesterol, triglycerides, alkaline phosphatase, aspartate aminotransferase, and alanine aminotransferase were measured on an automatic enzymatic analyzer. $\mathrm{HbA}_{1 \mathrm{c}}$ was determined by standard procedures. Insulin was measured by radioimmunoassay. The homeostasis model of assessment insulin resistance index was calculated. Plasma levels of apelin (human apelin-36) were assayed using a commercially available enzyme immunoassay kit (Phoenix Pharmaceuticals Inc, Burlingame, CA, USA) according to the manufacturer's instructions.

\section{Statistical analysis}

The results are presented as the mean \pm standard deviation or as a ratio. The Kolmogorov-Smirnov test was used to determine the distribution characteristics of variables and Levene's test was used to evaluate the equality of variance. Comparisons between and within groups were performed by independent-samples $t$-test and paired-samples $t$-test, respectively. All tests were two-tailed. The relationship between variables was analyzed by Spearman's rho correlation. Differences and correlations were considered to be statistically significant at $P<0.05$. Statistical Package for the Social Sciences version 17.0 software (SPSS Inc, Chicago, IL, USA) was used for the statistical analysis.

\section{Results}

In our study, 25 patients with T2DM treated by metformin (1,000 $\mathrm{mg} /$ day) alone were initially recruited. One patient was lost to follow-up and excluded from the statistical analysis. Patient characteristics and laboratory data for the study

Table I Characteristics and laboratory data for study participants

\begin{tabular}{|c|c|c|c|}
\hline & Metformin group $n=25$ & Control group $n=25$ & $P$-value \\
\hline Sex (male/female) & $17 / 8$ & $\mathrm{II} / \mathrm{I} 4$ & 0.087 \\
\hline Age (years) & $52.52 \pm 9.16$ & $52.84 \pm 9.34$ & 0.903 \\
\hline BMI $\left(\mathrm{kg} / \mathrm{m}^{2}\right)$ & $24.93 \pm 3.50$ & $25.06 \pm 2.60$ & 0.887 \\
\hline Waist (cm) & $91.75 \pm 10.81$ & $86.98 \pm 5.44$ & $0.08 I$ \\
\hline WHR & $0.93 \pm 0.07$ & $0.90 \pm 0.04$ & 0.125 \\
\hline Systolic BP (mmHg) & $122.96 \pm 17.46$ & $119.20 \pm 9.82$ & 0.355 \\
\hline Diastolic BP (mmHg) & $75.42 \pm 13.75$ & $80.68 \pm 5.57$ & 0.083 \\
\hline $\mathrm{HbA}_{\mathrm{Ic}}(\%)$ & $7.43 \pm 0.61$ & $5.73 \pm 0.24$ & $0.000 *$ \\
\hline $\mathrm{TC}(\mathrm{mmol} / \mathrm{L})$ & $4.55 \pm 0.78$ & $4.14 \pm 0.6 I$ & $0.048^{*}$ \\
\hline TG (mmol/L) & I.78 \pm 0.79 & $0.99 \pm 0.40$ & $0.000 *$ \\
\hline $\mathrm{HDL}-\mathrm{C}(\mathrm{mmol} / \mathrm{L})$ & $1.16 \pm 0.24$ & $1.40 \pm 0.30$ & $0.004^{*}$ \\
\hline LDL-C (mmol/L) & $2.79 \pm 0.58$ & $2.35 \pm 0.50$ & $0.007^{*}$ \\
\hline Apelin (ng/mL) & $6.09 \pm 4.90$ & $1.93 \pm 1.81$ & $0.000^{*}$ \\
\hline
\end{tabular}

Notes: Data presented as mean \pm standard deviation or as a ratio. ${ }^{*} P$-value $<0.05$.

Abbreviations: BP, blood pressure; BMI, body mass index; WHR, waist to hip ratio; $\mathrm{HbA}_{\mathrm{lc}}$, glycosylated hemoglobin; TG, triglycerides; HDL-C, high-density lipoprotein cholesterol; LDL-C, low-density lipoprotein cholesterol; TC, total cholesterol. 


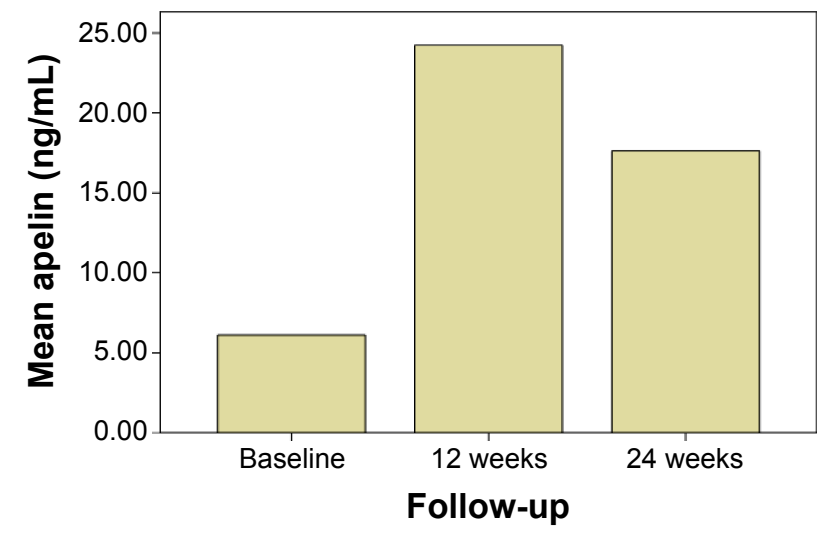

Figure I Comparison of apelin levels after vildagliptin treatment.

participants are shown in Table 1. The two groups did not differ in age, sex, BMI, waist, or blood pressure.

The plasma apelin level was significantly higher in the metformin group than in controls $(1.93 \pm 1.81 \mathrm{ng} / \mathrm{mL}$ versus $6.09 \pm 4.9 \mathrm{ng} / \mathrm{mL} ; P<0.05)$. After 12 weeks of add-on vildagliptin therapy, fasting blood glucose levels and $\mathrm{HbA}_{1 \mathrm{c}}$ were lower than at baseline. Apelin increased significantly by $18.15 \mathrm{ng} / \mathrm{mL}$ from baseline $(6.09 \pm 4.90 \mathrm{ng} / \mathrm{mL}$ versus $24.23 \pm 12.59 \mathrm{ng} / \mathrm{mL} ; P=0.029$ ). Patients were followed up to 24 weeks, by which time the apelin levels decreased, albeit not significantly $(24.23 \pm 12.59 \mathrm{ng} / \mathrm{mL}$ versus $17.62 \pm 8.51 \mathrm{ng} / \mathrm{mL}$; $P=0.975$ ). The apelin levels are shown in Figure 1. Other clinical and biochemical characteristics are shown in Table 2. No severe hypoglycemia events were observed. Other noteworthy adverse effects, such as cardiovascular events or heart failure, were not reported during the study period. We also searched for correlations between apelin and the other parameters, but found no correlation between blood apelin levels and BMI, lipid levels, or insulin resistance (Table 3).

\section{Discussion}

Apelin signaling may have an important role in the physiopathology of several diseases, including hypertension, heart failure, cardiovascular disease, T2DM, and obesity, although its effects and functions are still unclear. However, increasing evidence suggests that apelin is involved in regulation of multiple physiological functions, including food intake, blood pressure, and glucose utilization. ${ }^{14,15}$

Our study demonstrated a remarkable increase in apelin levels in response to treatment with metformin in T2DM when compared with healthy controls. A plausible explanation is that metformin triggers secretion of apelin via adenosine $5^{\prime}$ monophosphate-activated protein kinase, leading to alleviation of insulin resistance. ${ }^{16}$

This study demonstrates the positive effects of vildagliptin on plasma apelin levels in patients with T2DM, and is the first study to our knowledge to show a marked increase in apelin concentrations by add-on vildagliptin treatment. The effect was sustained until 24 weeks when compared with apelin levels at baseline. As we know, DPP-4 inhibitors inhibit DPP-4-dependent inactivation of glucose-dependent insulinotropic polypeptide (GIP) and glucagon-like peptide-1

Table 2 Clinical and biochemical parameters at baseline and at the end of the study

\begin{tabular}{|c|c|c|c|c|c|c|}
\hline & Baseline & I 2 weeks & 24 weeks & $P \mid n=25$ & $P 2 \mathrm{n}=24$ & $P 3 n=24$ \\
\hline $\mathrm{HbA}_{\mathrm{Ic}}(\%)$ & $7.43 \pm 0.61$ & $6.78 \pm 0.74$ & $6.82 \pm 0.73$ & $0.005^{*}$ & $0.008^{*}$ & 0.057 \\
\hline FPG (mmol/L) & $7.35 \pm 0.85$ & $6.53 \pm 0.76$ & $6.58 \pm 1.13$ & $0.037 *$ & $0.04 I^{*}$ & 0.12 \\
\hline FINS $(\mu \mathrm{U} / \mathrm{mL})$ & $7.74 \pm 4.38$ & $8.12 \pm 4.12$ & $9.04 \pm 4.08$ & 0.545 & 0.890 & 0.890 \\
\hline HOMA-\% B & $48.66 \pm 21.10$ & $56.43 \pm 26.32$ & $64.88 \pm 34.44$ & 0.656 & 0.481 & $0.48 I$ \\
\hline HOMA-IR & $1.29 \pm 0.72$ & $1.38 \pm 0.59$ & $1.47 \pm 0.66$ & 0.872 & 0.673 & 0.958 \\
\hline $\mathrm{TC}(\mathrm{mmol} / \mathrm{L})$ & $4.55 \pm 0.78$ & $4.78 \pm 0.82$ & $4.47 \pm 0.48$ & 0.812 & 0.465 & 0.873 \\
\hline TG (mmol/L) & $1.78 \pm 0.79$ & $2.03 \pm 0.67$ & $1.98 \pm 0.78$ & 0.954 & 0.115 & 0.665 \\
\hline HDL-C (mmol/L) & $1.16 \pm 0.24$ & $1.17 \pm 0.28$ & $1.18 \pm 0.29$ & 0.253 & 0.381 & 0.918 \\
\hline LDL-C (mmol/L) & $2.79 \pm 0.58$ & $2.95 \pm 0.72$ & $2.72 \pm 0.41$ & 0.330 & 0.901 & 0.845 \\
\hline $\mathrm{Cr}(\mu \mathrm{mol} / \mathrm{L})$ & $56.74 \pm I I .20$ & $57.58 \pm 15.46$ & $56.44 \pm 9.75$ & 0.232 & 0.203 & 0.270 \\
\hline UA $(\mu \mathrm{mol} / \mathrm{L})$ & $321.83 \pm 8.56$ & $312.00 \pm 116.01$ & $305.11 \pm 80.03$ & 0.375 & 0.547 & 0.123 \\
\hline $\mathrm{ALT}(\mathrm{U} / \mathrm{L})$ & $21.30 \pm 4.06$ & $22.08 \pm 4.40$ & $22.33 \pm 4.61$ & 0.950 & 0.069 & 0.869 \\
\hline AST (U/L) & $20.09 \pm 5.48$ & $21.92 \pm 5.42$ & $21.33 \pm 4.97$ & 0.403 & 0.064 & 0.509 \\
\hline ALP (U/L) & $75.17 \pm 23.54$ & $72.83 \pm 27.30$ & $77.22 \pm 22.93$ & 0.825 & 0.634 & 0.236 \\
\hline r-GT (U/L) & $31.74 \pm 15.72$ & $31.00 \pm 18.99$ & $30.33 \pm 16.29$ & 1.00 & 0.662 & 0.478 \\
\hline
\end{tabular}

Notes: Data presented as mean \pm standard deviation or as a ratio. $* P$-value $<0.05$.

Abbreviations: FPG, fasting plasma glucose; FINS, fasting serum insulin; Cr, creatinine; UA, uric acid; ALT, alanine aminotransferase; AST, aspartate aminotransferase; ALP, alkaline phosphatase; r-GT, r-glutamyl transferase; HOMA-\% B, homeostasis model assessment of steady-state beta cell function; HOMA-IR, homeostasis model assessment of insulin resistance; $P I, P$-values for changes in variables between baseline and follow-up at $I 2$ weeks; $P 2, P$-values for changes in variables between baseline and follow-up at 24 weeks; P3, P-values for changes in variables between follow-up at 12 weeks and 24 weeks; HDL-C, high-density lipoprotein cholesterol; LDL-C, low-density lipoprotein cholesterol; TG, triglycerides; TC, total cholesterol; $\mathrm{HbA}_{\mathrm{Ic}}$, glycosylated hemoglobin. 
Table 3 Correlations between plasma apelin levels and selected anthropometric and metabolic variables

\begin{tabular}{lll}
\hline & $\boldsymbol{r}$ & $\boldsymbol{P}$-value \\
\hline Age (years) & -0.092 & 0.669 \\
BMI $\left(\mathrm{kg} / \mathrm{m}^{2}\right)$ & 0.058 & 0.793 \\
Waist $(\mathrm{cm})$ & 0.082 & 0.811 \\
WHR & 0.219 & 0.518 \\
Systolic BP (mmHg) & 0.228 & 0.295 \\
Diastolic BP (mmHg) & 0.179 & 0.413 \\
HbA Ic $\left.^{2}\right)$ & -0.241 & 0.268 \\
TC (mmol/L) & 0.016 & 0.943 \\
TG (mmol/L) & 0.169 & 0.441 \\
HDL-C (mmol/L) & 0.309 & 0.151 \\
LDL-C (mmol/L) & -0.070 & 0.750 \\
HOMA-\% B & 0.249 & 0.276 \\
HOMA-IR & 0.116 & 0.608 \\
\hline
\end{tabular}

Abbreviations: $\mathrm{BP}$, blood pressure; $\mathrm{BMI}$, body mass index; WHR, waist to hip ratio; $\mathrm{HbA}_{\mathrm{Ic}}$, glycosylated hemoglobin; TG, triglycerides; HDL-C, high-density lipoprotein cholesterol; LDL-C, low-density lipoprotein cholesterol; TC, total cholesterol; HOMA-\% B, homeostasis model assessment of steady-state beta cell function; HOMA-IR, homeostasis model assessment of insulin resistance.

(GLP-1), thereby enhancing their biological actions.${ }^{17}$ Recent research suggests an interplay between apelin and GLP-1. ${ }^{18}$ GLP-1 mediates the enteric and/or systemic action of apelin, and apelin also stimulates the secretion of the incretin GLP-1 both in vitro and in vivo. Wattez et al suggested that apelin might modulate digestive functions, food intake behavior, and glucose homeostasis via apelin-induced release of enteric cholecystokinins and also via incretin-releasing activity on enteric GLP-1. ${ }^{18}$ This implies that the increase in plasma apelin levels associated with vildagliptin therapy might be related to increased GLP-1. The GIP receptor is expressed in adipose tissues, ${ }^{19}$ and GIP plays a critical role in accumulation of fat. ${ }^{20}$ These observations suggest that GIP had an effect on adipocytes, but there have been no reports published on the relationship between GIP and apelin. It is not clear that whether the vildagliptin-induced improvement in apelin levels is related to GIP. At present, the specific mechanism of the effect of vildagliptin on apelin levels is not understood, but may be related to GLP or GIP. In order to verify this hypothesis, further study of the relationship between apelin and GLP or GIP in humans is needed.

In mice, apelin was shown to act on the intestine through a luminal action to control glucose absorption. ${ }^{21}$ In addition, apelin is expressed in brain regions implicated in food and water intake. One study suggested that apelin was involved in the central control of feeding. ${ }^{22}$ Therefore, we speculate that inhibition of appetite by vildagliptin might be related to increased apelin levels.

We did not find any relationship between apelin and BMI in T2DM or isolated hypercholesterolemia. This implies that the association between circulating apelin and BMI might be limited to overt obesity. Adiposity may not be a major determinant in some conditions, and different mechanisms might be involved in regulation of blood apelin concentrations. No significant relationship was found between apelin levels and insulin sensitivity in patients with T2DM in our research. It differs from previous studies probably because subjects in our research had received metformin therapy. This finding suggests that the association between insulin and apelin might not always be in the same direction under physiological or pathological conditions.

Our present study has several limitations. Because of the narrow selection criteria, the sample size was small. Hence, our data may not be representative for all subjects with T2DM. Another limitation was the use of the homeostasis model of assessment for insulin resistance index instead of the euglycemic hyperinsulinemic clamp method. The former is a less accurate method, and mainly reflects hepatic insulin sensitivity. Further larger studies are required in patients with and without vascular complications or retinopathy to investigate the role of apelin in T2DM and the effects of antidiabetic drugs on plasma levels of apelin.

\section{Conclusion}

In conclusion, vildagliptin and metformin had a favorable effect on apelin levels and improved glycemic control. Combined treatment with metformin plus vildagliptin was superior to metformin monotherapy in terms of modifying apelin levels in patients with T2DM. This clinically important finding may be attributed to the complementary modes of action of vildagliptin and metformin. Addition of vildagliptin may be useful for patients inadequately controlled on a low dose of metformin.

\section{Acknowledgment}

This study was funded by a grant from Shanghai Science and Technology Development (114119b0400).

\section{Disclosure}

The authors report no conflicts of interest in this work.

\section{References}

1. Tatemoto K, Hosoya M, Habata Y, et al. Isolation and characterization of a novel endogenous peptide ligand for the human APJ receptor. Biochem Biophys Res Commun. 1998;251(2):471-476.

2. Sorhede Winzell M, Magnusson C, Ahren B. The APJ receptor is expressed in pancreatic islets and its ligand, apelin, inhibits insulin secretion in mice. Regul Pept. 2005;131(1-3):12-17.

3. Ringstrom C, Nitert MD, Bennet $\mathrm{H}$, et al. Apelin is a novel islet peptide. Regul Pept. 2010;162(1-3):44-51. 
4. Habchi M, Duvillard L, Cottet V, et al. Circulating apelin is increased in patients with type 1 or type 2 diabetes and is associated with better glycaemic control. Clin Endocrinol. 2014;81(5):696-701.

5. Yue P, Jin H, Aillaud M, et al. Apelin is necessary for the maintenance of insulin sensitivity. Am J Physiol Endocrinol Metab. 2010; 298(1):E59-E67.

6. Zhu S, Sun F, Li W, et al. Apelin stimulates glucose uptake through the PI3K/Akt pathway and improves insulin resistance in 3T3-L1 adipocytes. Mol Cell Biochem. 2011;353(1-2):305-313.

7. Xu S, Han P, Huang M, et al. In vivo, ex vivo, and in vitro studies on apelin's effect on myocardial glucose uptake. Peptides. 2012;37(2): 320-326.

8. Castan-Laurell I, Dray C, Knauf C, Kunduzova O, Valet P. Apelin, a promising target for type 2 diabetes treatment? Trends Endocrinol Metab. 2012;23(5):234-241.

9. Erdem G, Dogru T, Tasci I, Sonmez A, Tapan S. Low plasma apelin levels in newly diagnosed type 2 diabetes mellitus. Exp Clin Endocrinol Diabetes. 2008;116(5):289-292.

10. Zhang Y, Shen C, Li X, et al. Low plasma apelin in newly diagnosed type 2 diabetes in Chinese people. Diabetes Care. 2009;32(12):1146.

11. Shestakova MV, Suhareva Olu, Chernova TO, et al. [A combination of dipeptidyl peptidase-4 inhibitor and metformin in the treatment of patients with type 2 diabetes mellitus: effective control of glycemia, weight, and quantitative body composition]. Ter Arkh. 2013; 85(8):49-55. Russian.

12. Wang J, Ciaraldi TP, Samad F. Tissue factor expression in obese type 2 diabetic subjects and its regulation by antidiabetic agents. $J$ Obes. 2015;2015:291209.

13. Shimasaki T, Masaki T, Mitsutomi K, et al. The dipeptidyl peptidase-4 inhibitor des-fluoro-sitagliptin regulates brown adipose tissue uncoupling protein levels in mice with diet-induced obesity. PLoS One. 2013;8(5):e63626.
14. Reaux A, De Mota N, Skultetyova I, et al. Physiological role of a novel neuropeptide, apelin, and its receptor in the rat brain. JNeurochem. 2001;77(4): 1085-1095.

15. Reaux A, Gallatz K, Palkovits M, Llorens-Cortes C. Distribution of apelin synthesizing neurons in the adult rat brain. Neuroscience. 2002; 113(3):653-662.

16. Kadoglou NP, Tsanikindis H, Kapelouzou A, et al. Effects of rosiglitazone and metformin treatment on apelin, visfatin, and ghrelin levels in patients with type 2 diabetes mellitus. Metabolism. 2010;59(3): 373-379.

17. Yutaka S, Daisuke Y. Glucose-dependent insulinotropic polypeptide and glucagon-like peptide-1: incretin actions beyond the pancreas. J Diabetes Investig. 2013;4(2):108-130.

18. Wattez JS, Ravallec R, Cudennec B, et al. Apelin stimulates both cholecystokinin and glucagon-like peptide 1 secretions in vitro and in vivo in rodents. Peptides. 2013;48:134-136.

19. Yip RG, Boylan MO, Kieffer TJ, Wolfe MM. Functional GIP receptors are present on adipocytes. Endocrinology. 1998;139(9):4004-4007.

20. Miyawaki K, Yamada Y, Ban N, et al. Inhibition of gastric inhibitory polypeptide signaling prevents obesity. Nat Med. 2002;8(7):738-742.

21. Dray C, Sakar Y, Vinel C, et al. The intestinal glucose-apelin cycle controls carbohydrate absorption in mice. Gastroenterology. 2013;144(4) 771-780.

22. O'Shea M, Hansen MJ, Tatemoto K, Morris MJ. Inhibitory effect of apelin -12 on nocturnal food intake in the rat. Nutr Neurosci. 2003 ; 6(3):163-167.
Drug Design, Development and Therapy

\section{Publish your work in this journal}

Drug Design, Development and Therapy is an international, peerreviewed open-access journal that spans the spectrum of drug design and development through to clinical applications. Clinical outcomes, patient safety, and programs for the development and effective, safe, and sustained use of medicines are a feature of the journal, which

\section{Dovepress}

has also been accepted for indexing on PubMed Central. The manuscript management system is completely online and includes a very quick and fair peer-review system, which is all easy to use. Visit http://www.dovepress.com/testimonials.php to read real quotes from published authors.

Submit your manuscript here: http://www.dovepress.com/drug-design-development-and-therapy-journal 